\title{
Remotely Piloted Aircraft Systems and a Wireless Sensors Network for Radiological Accidents
}

\author{
A. Reyes-Muñoz, ${ }^{1}$ J. Guerrero-Ibáñez, ${ }^{2}$ E. Pastor, ${ }^{1}$ M. Gasull, ${ }^{1}$ and C. Barrado ${ }^{1}$ \\ ${ }^{1}$ Computer Architecture Department, BarcelonaTech, Castelldefels, Spain \\ ${ }^{2}$ School of Telematics, University of Colima, Colima, COL, Mexico \\ Correspondence should be addressed to A. Reyes-Muñoz; mreyes@ac.upc.edu
}

Received 1 July 2016; Accepted 20 September 2016

Academic Editor: Hikmat Asadov

Copyright ( 2016 A. Reyes-Muñoz et al. This is an open access article distributed under the Creative Commons Attribution License, which permits unrestricted use, distribution, and reproduction in any medium, provided the original work is properly cited.

In critical radiological situations, the real time information that we could get from the disaster area becomes of great importance. However, communication systems could be affected after a radiological accident. The proposed network in this research consists of distributed sensors in charge of collecting radiological data and ground vehicles that are sent to the nuclear plant at the moment of the accident to sense environmental and radiological information. Afterwards, data would be analyzed in the control center. Collected data by sensors and ground vehicles would be delivered to a control center using Remotely Piloted Aircraft Systems (RPAS) as a message carrier. We analyze the pairwise contacts, as well as visiting times, data collection, capacity of the links, size of the transmission window of the sensors, and so forth. All this calculus was made analytically and compared via network simulations.

\section{Introduction}

Radiation monitoring is an essential part of any radiation protection program of a nuclear plant. The measurement of ambient values is crucial to minimize exposure to workers and estimates occupancy times for radiological areas. In that scenario, aerial surveys are useful to provide a precise perspective for monitoring, and, in case of an accident, it would not require human participation directly into potential hot zones to get data on the leak scope.

For these kinds of emergency situations, the Remotely Piloted Aircraft Systems (RPAS) have been widely proposed, basically to obtain aerial images [1-6]. In the specific case of a radiological incident, the use of the RPAS as message carriers is a very attractive solution to gather information of potentially dangerous large areas of terrain due to the altitude they can reach.

The use of RPAS as a relay system, acting as carrier of data retrieved from ground sensors, has been studied in a list of previous works [7-11], for both statically allocated sensors and mobile ground terminals. Another approach is setting the sensors in a fleet of RPAS which collaborate in gathering data named flying ad hoc networks or FANETs $[12,13]$. Choi et al. focus more on the RPAS route and the energy-efficient communication ground/air using a single RPAS [14].

In our proposal there is a Wireless Sensor Network (WSN) composed of a set of distributed wireless sensors located on the disaster area, several ground vehicles, and a RPAS.

The RPAS has a previously defined flight plan that indicates all the waypoints that the RPAS has to fly over. Located physically in each logical waypoint there are one or several sensors which are in charge of collecting radiological properties of the air and soil in the surrounding environment and convert these properties into electrical signals. So when the RPAS flies through the waypoints it picks up all the information from sensors and sends it to the control center which is remotely located.

The ground vehicles are mobile distributed sensors that collect data from their environment and try to communicate the data to the RPAS. As ground vehicles collect information from different sensors they have higher priority than fixed sensors when a RPAS flies over the area. So when a ground vehicle appears in the RPAS transmission range then the RPAS collects all stored information by the ground vehicle and reconfigure its own flight plan in order to arrive on 
time to achieve communication with most of the other sensors during its active phase. This means that the sensor transmission window has to be increased or decreased in real time and also the speed of the RPAS is readjusted to achieve in this way a greater amount of data collection collected by sensors and vehicles.

All calculations were mathematical and compared with a network simulation. Finally, we developed a set of interfaces to simulate the geographical area of the nuclear plant in which the RPAS follows the flight plan to gather the sensors data.

The paper is organized as follows: Section 2 describes the scenario that is simulated in the Asco nuclear plant in case a radiological accident occurs. Section 3 is dedicated to explain the components of our WSN. Section 4 shows the test that we have done in terms of network architecture and transmission protocols, capacity analysis of the links, and the implementation of the system interfaces. Finally, Section 4 describes the results of the research. Section 5 has a discussion and Section 6 concludes the paper.

\section{Ascó Nuclear Plant}

Ascó is a nuclear plant located in the town of Ascó in the province of Tarragona [15]. Ascó has a control center that works as a reactive entity where the operating supervisor and senior operating personnel operate and monitor major plant equipment which provides alarms and notification in case any problem arises.

A map of specific actions is activated for the three government regulated predefined areas [15]:

(i) Zone 0, area under operator control: it is an area with a $750 \mathrm{~m}$ radius. Emergency actions are defined in the emergency plan of the nuclear plant.

(ii) Zone 1, area that requires urgent protection measures: it is a concentric circle with a $10 \mathrm{~km}$ radius including area 0 . The radiation exposure may be in both the atmosphere and soil.

(iii) Zone 2, area that covers 30 concentric kilometers: radioactivity is usually on objects that are on the ground.

In this sense we propose a Wireless Sensor Network (WSN) that is consistent with the current plan for nuclear exercises of Ascó. If a sensor reading in the vicinity of the nuclear plant exceeds a preset radiological upper limit for the environment, then an alarm immediately notifies the control center.

\section{Deployment of a Wireless Sensor Network}

There are several crucial aspects in developing a WSN for radiological environments, such as the following: (a) Long expected network lifetime should be considered to reduce human intervention, for example, for batteries replacement.

(b) The WSNs for harsh environments have to contemplate the fact that node failures may occur unexpectedly, so synchronization and routing algorithms need to be fault tolerant to guarantee network robustness. (c) There is a tradeoff between energy consumption and monitoring capabilities.

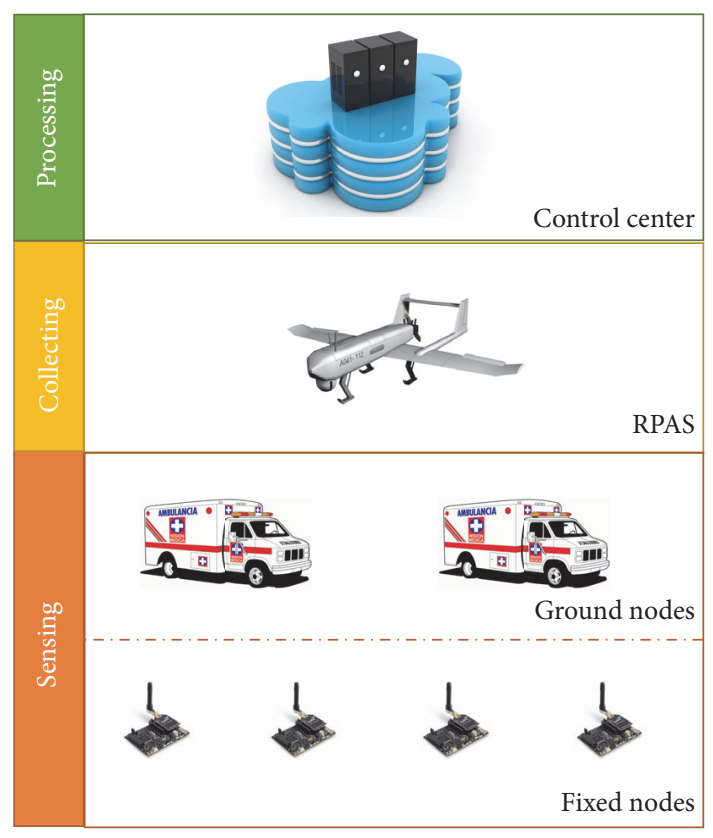

FIgURE 1: Three-layer model.

(d) The gradual accumulation of radiation effects on the WSN over a long period of time is known as displacement damage (displacement of atoms from their original position in the lattice sites) and damage due to total ionizing dose (absorption of energy by electronic ionization in the insulators) [16]. The mitigation approaches are mainly based on radiation hardened microelectronic techniques [17] and radiation shielding used to enclose the sensors with highly dense materials such as lead and cadmium.

For our proposal we designed a three-layer model with four different components (see Figure 1).

Sensing layer contains all devices that gather environmental data. It is formed by two components. First component is the set of distributed fixed sensors that are able to communicate with the RPAS via a highly reliable and secure link. These are the basic sensor nodes which are responsible for information collection. Each wireless sensor node is a complex device that embeds a microcontroller, an SD memory card, GPS, an accelerometer, a temperature sensor, and specific sensors for radiation levels and gases [18]. The sensors collect radiological properties of the air and soil and convert these properties into electrical signals. The transceiver is the RF module responsible for wireless communication with the RPAS. Technology used for data transmission (either the RPAS or a grand vehicle) is XBee 802.15.4 $2.4 \mathrm{GHz}$ with a range of 500 meters and bit rate of $250 \mathrm{kbps}$. The ZigBee protocol follows the IEEE 802.15.4 standard for WSN employing low data rates requirements and security services based on a 128-bit AES algorithm added to the security model provided by IEEE 802.15.4 [19]. We establish the theoretical positions of the waypoints (physical position in the map that allows the RPAS to fly over the sensors) because due to the harsh conditions of the terrain 
sometimes it is not possible to place sensors on the exact coordinates.

Second component is the ground vehicles (emergency units, police, firefighters, etc.) which are considered mobile nodes in the WSN. These vehicles are equipped with longrange radios, allowing collecting information of sensors within their coverage range. These kinds of nodes have higher priority level than fixed sensors and they can perform various functions such as data compression and fusion. Each ground vehicle can be used as a gateway to enhance the connectivity with the RPAS and to reduce the amount of energy spent by sensor nodes in the data transmission process to RPAS. For that, ground vehicles contain a fixed amount of buffer memory, which is used to hold the collected sensing information until it transfers it to the RPAS when it arrives within its transmission range. The RPAS compares sensor IDs with the information of sensor included in the flight plan and then eliminates the sensors that have been collected already. Finally, the flight plan is rescheduled in real time and the transmission window is reconfigured in order to receive the collected information of ground vehicle.

Collecting layer is composed of the RPAS which is responsible for retrieving the sensed and stored information by ground vehicles and sensors and for delivering that information to the control center of the nuclear plant. We use a RPAS Sniper of Alpha Unmanned Systems widely used in urban environments [20] and enable speeds up to $150 \mathrm{~km} / \mathrm{h}$, altitude of $3000 \mathrm{~m}$, and a range of approximately 2 hours because of its low weight $14 \mathrm{~kg}$., $1.6 \mathrm{~m}$, and reduced size. The RPAS follows a predefined flight plan that indicates the position of the waypoints and periodically broadcasting its own location using a long-range radio. So when a RPAS detects a sensor or ground vehicle within its transmission range, it sends a communication request. The RPAS makes proactive movement (adjust speed and altitude) to meet with fixed wireless sensors that are distributed on the ground and ground vehicles that move randomly.

Finally, processing layer is composed by the control center which is responsible for processing the data received by the RPAS. This entity specifies the flight plan (with all the waypoints to be overflown by the RPAS) based on specific mission requirements.

3.1. Network Links. We define 4 links that combine different communication ranges and different requirements regarding their transmission window: (1) sensor-RPAS, (2) sensorground vehicle, (3) ground vehicle-RPAS, and (4) RPAScontrol center [21]. See Figure 2.

The first link has a temporal pattern of periodic motion, the second and third link are completely spontaneous, and the last link has an aperiodic pattern.

The data transmission protocol implements five steps: invitation to the network, data transfer, data management, data resend, and close communication. These five steps are implemented in the four mentioned links.

Nodes can be in one of three possible modes (see Figure 3).

Linked to the network, the node can accept requests to become part of the network. In transmission mode, the node

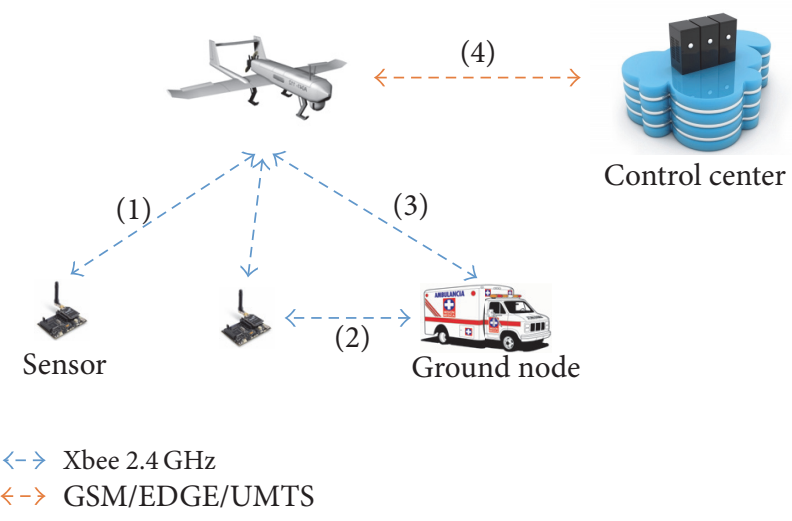

FIGURE 2: Network links.

reads the sensors and transmits the data to the RPAS or ground node. In case of ground vehicles, the transmission mode includes the time dedicated to gather data of neighbor nodes that appears in their transmission range. Disconnected mode is only for fixed networks, the radio interface is turned off, and neither transmission nor reception is possible.

A node is initially disconnected from the network; it means that node has not requested data transmission; during that time the sensor is sensing or resting to save energy.

When the node is on linked mode it can receive a request message from the RPAS or ground node and change to the transmission mode. After interaction with the RPAS or ground node, the node is disconnected from the network.

The RPAS operating modes are idle and working. Initially the RPAS is in idle mode. When RPAS detects a node (fixed node or ground node), a Link_REQ is sent from RPAS to the detected node. Upon receiving the service response, the RPAS changes to working mode to receive the sensed data. See Algorithm 1.

\section{Experimental Section}

In the experiments we used an XBee $(250 \mathrm{Kbps})$ sensor configured with a frame time of $10 \mathrm{~ms}$. The RPAS works as a client and downloads files located in sensors.

The initial window size is set to $64 \mathrm{~KB}$, which is the default window in most operating systems. The minimum time that will be taken for the ACK (acknowledgment) to arrive after the first package is sent (delay in the Xbee) is about four times the frame time, which in this case is RTT $\sim 4 * 10 \mathrm{~ms}=40 \mathrm{~ms}$. Thus, 512 kbits $(64 \mathrm{~KB})$ can be sent every $40 \mathrm{~ms}$. Therefore, the maximum throughput is $512 \mathrm{kbits} / 40 \mathrm{msg}=12.8 \mathrm{Mbps}$.

In the analysis of the sensor network we consider two types of variables.

The contact time is the time in which a pair of nodes are within their coverage area and therefore within reach of communicating with each other. The contact time influences the capacity of the network by limiting the amount of data that can be transferred between nodes.

The time between contacts is the time between two contacts, starting from the last contact with a node to the beginning of contact with another one. The time between 


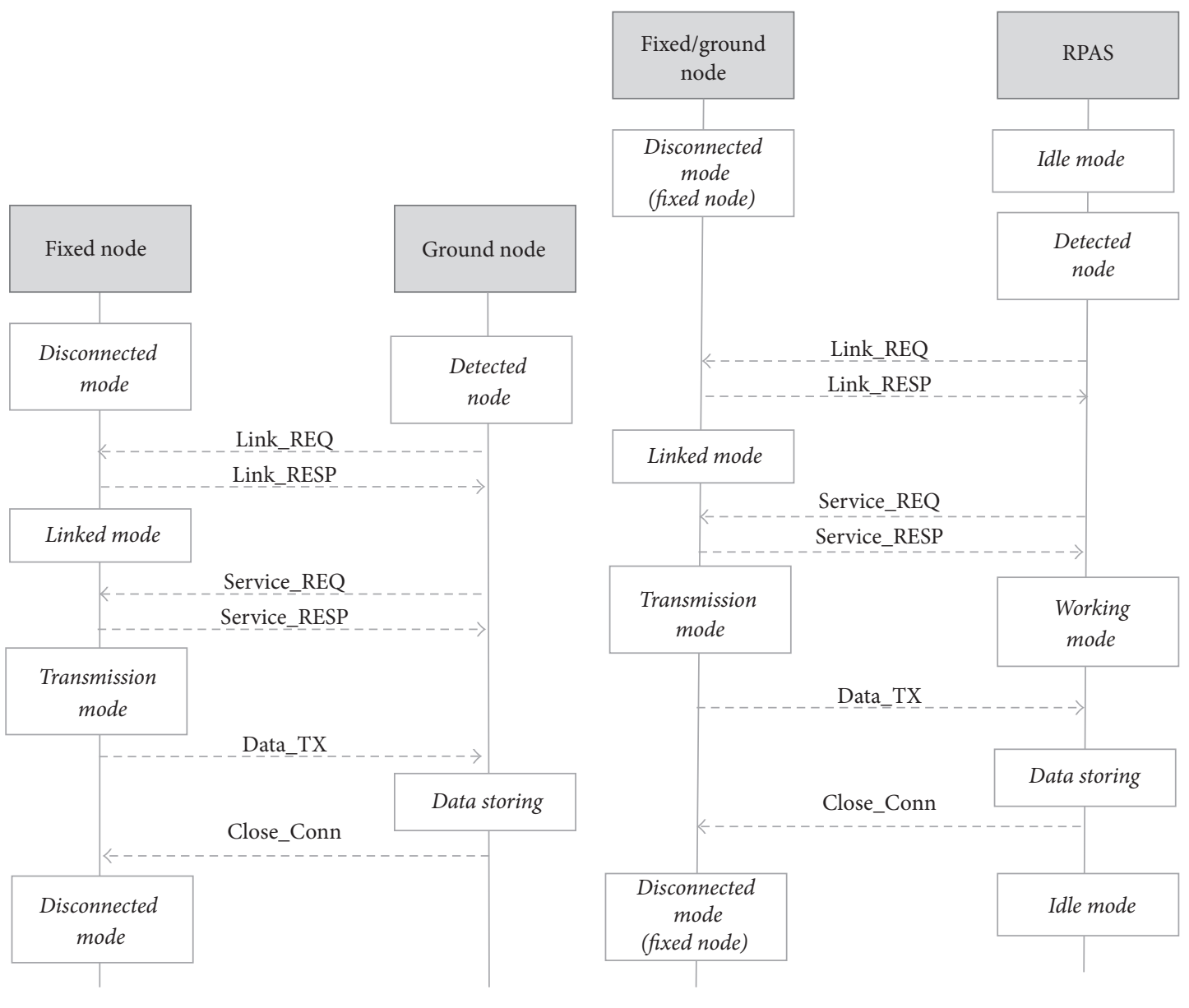

(a)

(b)

FIGURE 3: Operating modes: (a) fixed-ground and (b) fixed/ground and RPAS.

$H$ : the set of nodes requesting service

$P$ : Route of waypoints for the RPAS

If the RPAS is in idle mode

$H=$ empty set

The RPAS flights over the default flight plan

The RPAS periodically distributes location messages

If the UAV receive a service request message from a node $i$

Add $i$ to $H$

Calculate the new route of waypoints $P$

Set working mode to the RPAS

Reduce the RPAS speed over waypoints to receive the maximum amount of data

If transmission ends

Remove $I$ from $H$

If $H$ is not empty

Calculate new route $P$ for RPAS

Otherwise

RPAS Mode $=$ idle

Algorithm 1: RPAS modes. 


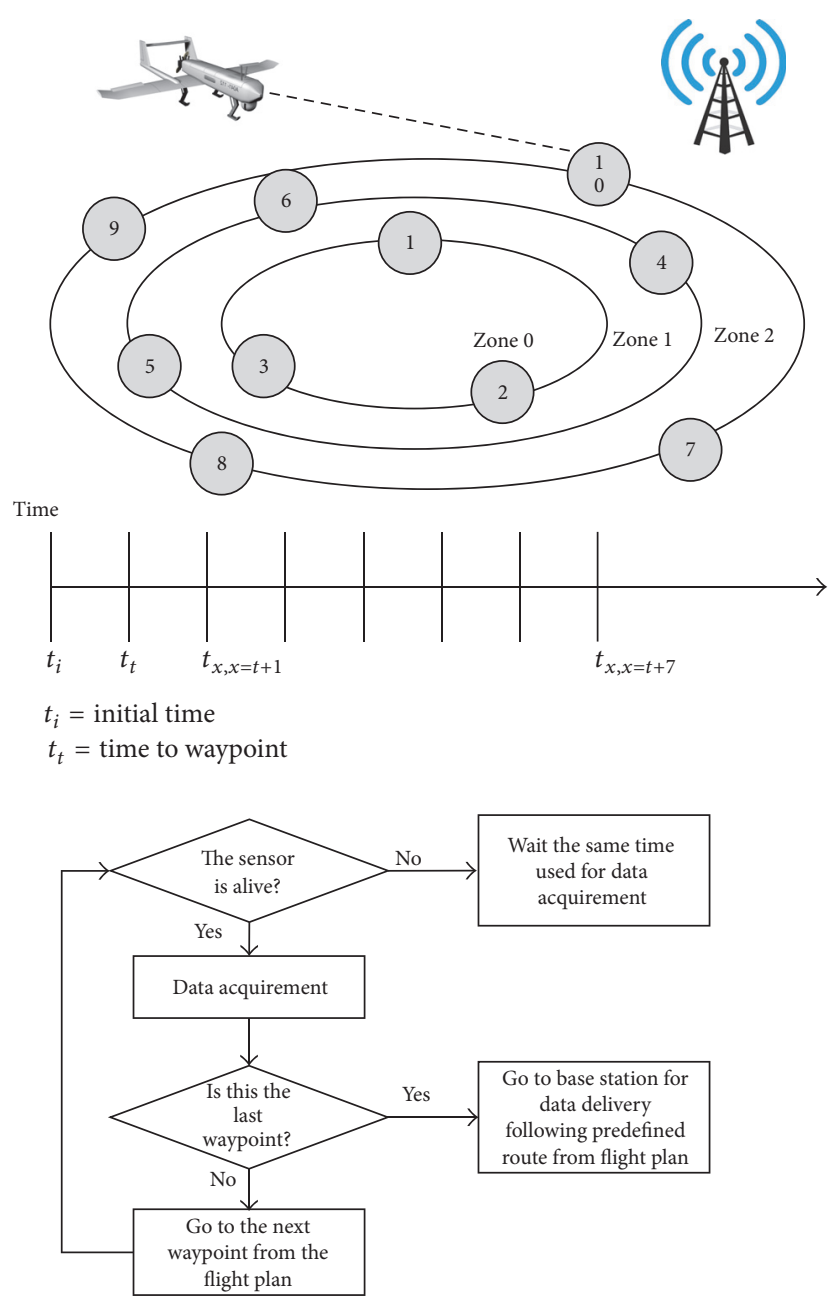

FIGURE 4: RPAS-sensor link.

contacts has an impact on network availability because it affects (a) the number of times that the transmission window of a sensor is opened when the RPAS flies over its position and (b) the frequency of opportunities a ground vehicle can transfer messages to the RPAS.

For case (a) the RPAS visits sensors in a predefined time to either send or receive information that will be transmitted or that comes from the control center. See Figure 4.

Assuming the RPAS flies at a constant speed and defining $\left\{w_{0}, w_{1}, \ldots, w_{n}\right\}$ as the set of waypoints contained in the flight plan, the total time required for the RPAS to visit all the waypoints is total time $=\sum_{i=0}^{n} t\left(w_{i}, w_{i+1}\right)$, where $t\left(w_{i}, w_{i+1}\right)$ represents the contact time required for the RPAS to collect data at the point $w_{i}$, plus the time between contacts you need to get from point $w_{i}$ to consecutive waypoint $w_{i+1}$.

In case (b) the contact time of the RPAS to collect information on ground vehicles is $t_{2}\left(G_{i}, D_{G}\right)$.

$t_{2}$ is the contact time required to collect data from a ground vehicle $G_{i}$ plus the contact time required for the RPAS to get close to the ground vehicle $D_{G}$. Therefore, $\sum_{i=0}^{n-1} t\left(w_{i}, w_{i+1}\right)$.

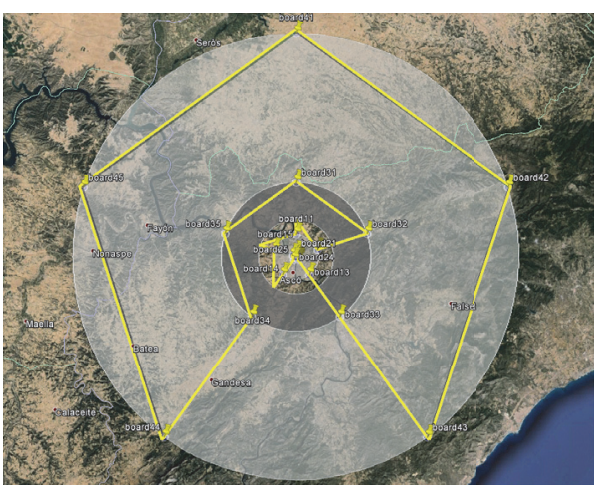

FIGURE 5: Flight plan 1. RPAS flies all the waypoints.

Therefore, the RPAS flies over a waypoint for a total time $/ w_{i}$ to get all the sensed and stored information from sensors and ground vehicles.

As mentioned above, the transmission window is initialized to the minimum size in order to save as much as possible of the sensor battery. Suppose the time of the transmission window is denoted as TimeW:

$$
\begin{gathered}
\text { TimeW } \geq \text { Time }_{\text {establishment }}+\text { Time }_{\text {transmission }} \\
+ \text { Time }_{\text {Close_comm }},
\end{gathered}
$$

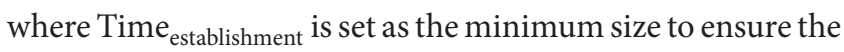
transmission opportunity and Time transmission $_{\text {is the required }}$ time to transfer all the stored information in the sensor to the RPAS.

Time $_{\text {Close_comm }}$ is the sensor's required time for closing the connection. Finally, the RPAS sends an ACK to the sensor indicating the next period of visit.

If $t_{2}>$ Time $W$ then it is necessary to adjust the speed of the RPAS or skip a visit to one or more waypoints from those indicated in the flight plan in order to be on time to achieve the maximum number of sensors set in linked mode (open transmission window).

The information sensed by ground vehicles has higher priority than information from fixed sensors, so the RPAS cannot ignore the communication of a ground vehicle.

In order to evaluate the performance of the proposal, two RPAS flight plans were defined.

(a) Flight plan 1 covers all the preestablished waypoints. We consider the scenario where the RPAS did not detect any ground node in the flight plan (See Figure 5); (b) flight plan 2 does not cover all waypoints due to data collection of ground vehicles (See Figure 6).

In flight plan 2, the RPAS speed is readjusted to arrive on time to the rest of preestablished waypoints. In this case we evaluate the transmission window size.

Figure 7 shows the size of the transmission window in both flight plans.

4.1. Sensor Network Interface. The interface that manages the sensor network is part of the mission monitor of ISIS+, a software we developed as part of the simulator for unmanned aerial systems [22]. The interface is linked to the simulator as 


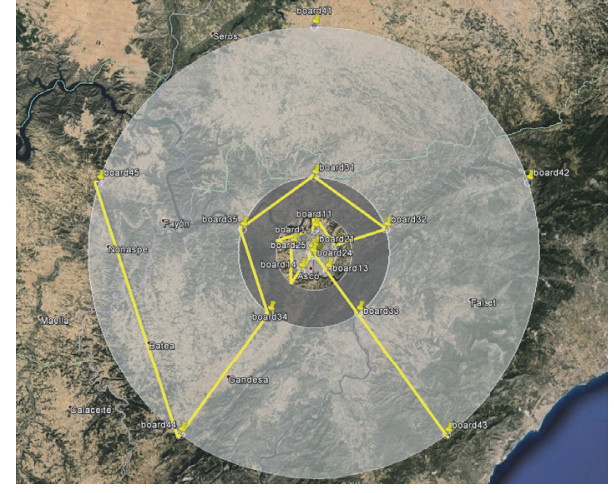

FIgURE 6: Flight plan 2. The RPAS does not visit all the waypoints.

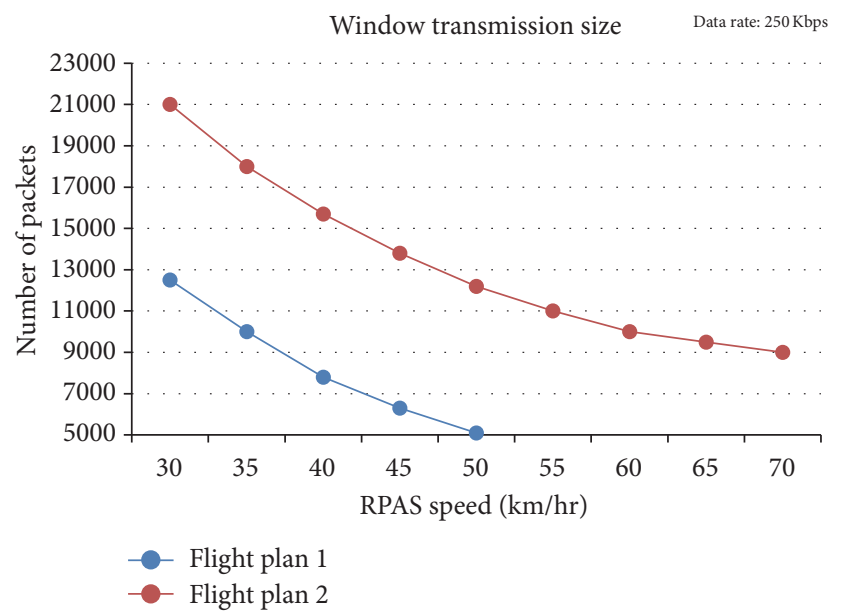

FIgURE 7: Transmission window size.

a service that is managed by our Middleware Architecture for embedded remote applications.

The interface has 4 menus: an Actuation Map to configure the number of sensors of Zones I, II, and III of the nuclear central. Sensor Manager is used to establish the sensor mode (linked or disconnected). The Flight Plan is the menu that generates the waypoints location, the RPAS speed, and total distance of the flight plan, among other parameters. A Communication Menu allows authorized users to modify the information of the flight plan and visualize the collected information by the RPAS as shown in Figure 8 .

\section{Results}

In order to evaluate the performance of our experiments, we ran several simulations in Castalia [23], a discrete-event simulator developed in $\mathrm{C}++$ based on the Omnet Framework [24] and used in similar studies [25]. The simulation model is based on a set of 50 replications. Each replication represents the simulation of a flight plan for data recollection.

The proposed scenario defined into Castalia is based on the sensors network designed to Ascó nuclear plant, which was explained in previous sections. The total number of sensors used for the simulation was 24 , which were

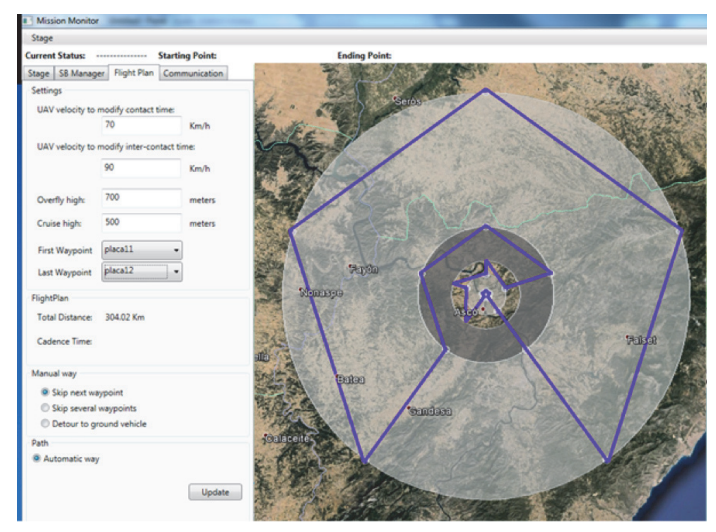

Figure 8: Sensor network interface.

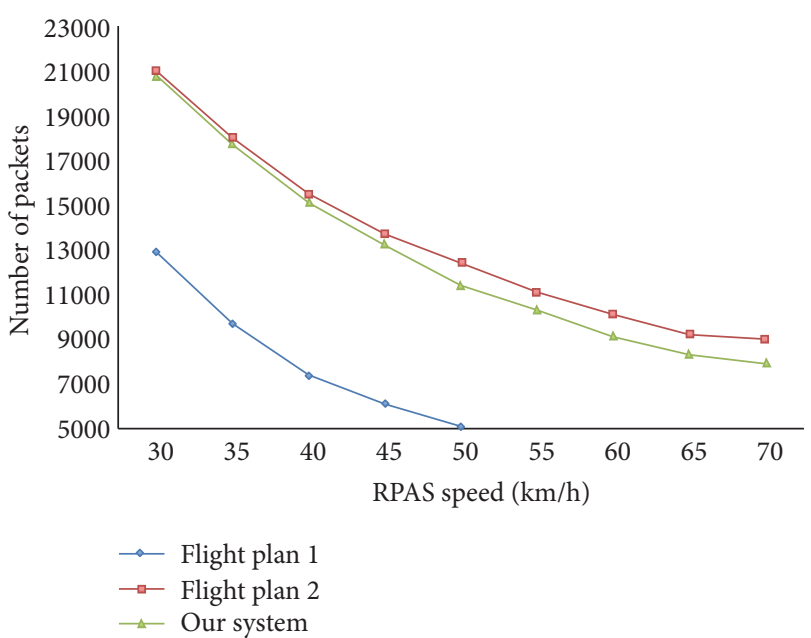

Figure 9: Transmission window size.

distributed in each zone as shown in Figure 5. We used different number of ground vehicles. Ground vehicles were uniformly distributed over the total monitoring area. The speed of ground vehicles was set to $15 \mathrm{~km} / \mathrm{h}$.

We evaluated the transmission window size and delivery ratio applying different speeds for RPAS to show the performance in the maximization of collected information. Additionally, we evaluated the cadence time in a flight plan that consider random ground vehicles.

Figure 9 shows the transmission window size obtained in the simulation of the two flight plans previously explained and evaluated analytically. When the speed is low we can observe a similar behavior of the results obtained in both analytical and simulated forms. However, as displayed, when the speed of the RPAS increases, our system in the simulation obtained values of transmission windows less almost $12 \%$ in comparison with the analytic model. When we analyzed the results obtained in the simulation model we observed that there is interference in the transmission process that is produced by the neighbor nodes which result in a difference between the analytical and the simulated models.

Figure 10 shows how the delivery ratio obtained in the simulation of the two flight plans previously explained varies 


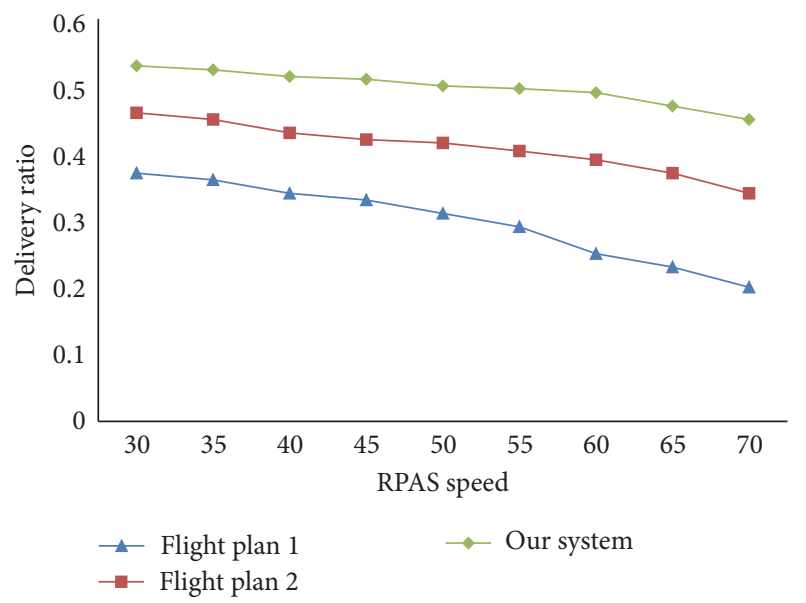

FIgURE 10: Delivery ratio.

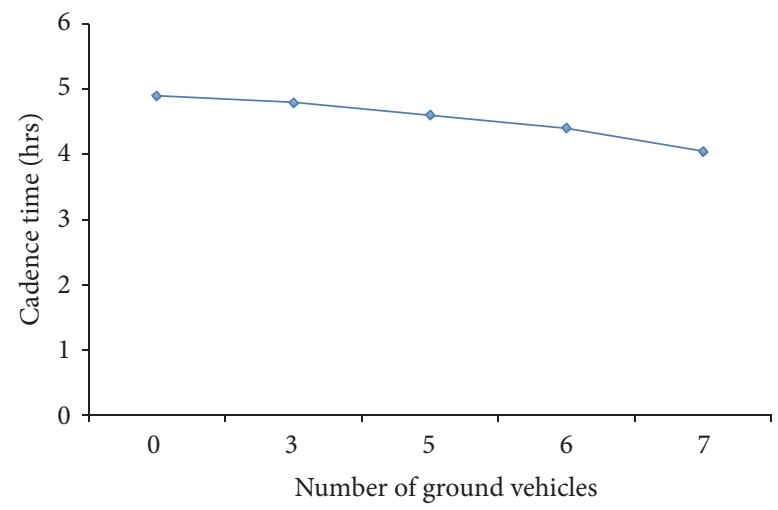

FIgURE 11: Cadence time.

firstly increasing and then decreasing slightly as the RPAS speed is increased. We observed when a constant speed is applied during all flight (flight plan 1) the delivery ratio suffers a decrement up to $48 \%$. On the other hand, when the RPAS is constant and ground vehicles are used, the delivery ratio increases up double with respect to flight plan 1 . When we use an adaptable speed, the delivery ratio increases up 32\%. The main reason behind this behavior is that when the RPAS speed increases, less packets can be delivered from sensor nodes because there is not sufficient time for the RPAS to collect packets from the nodes and the delivery ratio goes down; however, when the RPAS speed is adapted there is sufficient time for the RPAS to collect packets from the nodes and the delivery ratio can be kept.

Figure 11 shows the cadence time of the simulation. We analyze the results increasing the number of ground vehicles. The zero value in the number of vehicles represents the situation where the RPAS covers all the preestablished waypoints. We can observe that when the number of vehicles increases the system reduces the cadence time almost $17 \%$ in comparison with the complete coverage of the RPAS. As the priority of ground vehicles is higher than the one in the fixed sensors, if a ground vehicle is in the transmission range of the RPAS it will interfere with the established flight plan and with

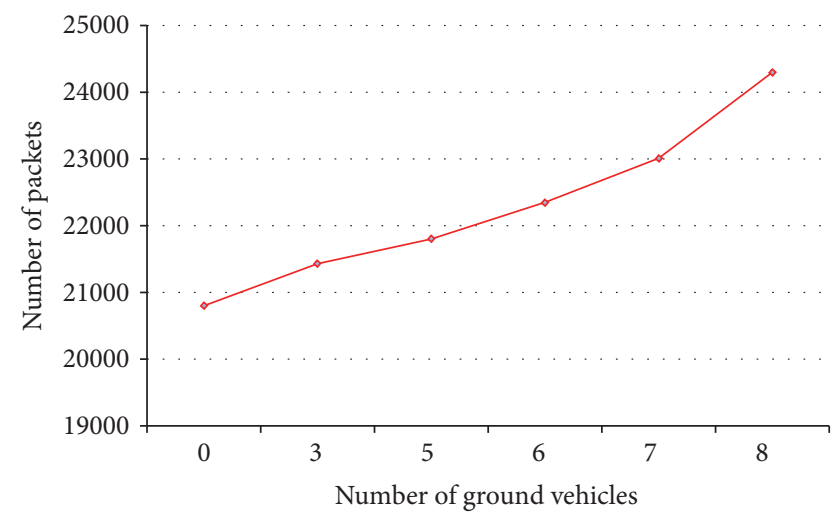

FIgURE 12: Transmission window size.

the adjustments the RPAS does not cover all the predefined waypoints. So the total distance is smaller given the reduction in the cadence time.

Finally, we evaluated the impact that the number of ground vehicles has in the collected data. Figure 12 shows an improvement of almost $17 \%$ in transmission window when the number of ground vehicles increases compared with the scenario where the RPAS covers all waypoints.

\section{Discussion}

In this article, we have focused on the use of a single carrier message (in the form of a RPAS) to provide communication between the nuclear plant and the control center with both fixed and mobile sensors.

However, a containment transmission can occur when multiple sensors of the same waypoint attempt communication with the RPAS, or when simultaneously a ground vehicle is in the transmission range of the RPAS.

Future work will analyze the network performance with multiple RPAS and cooperative routing protocols as we consider that multiple carriers' messages can potentially improve network capacity.

In this article we consider only the difference in priorities between the data provided by ground vehicles (highest priority) and sensor data.

Future work will differentiate the priority level of the sensors depending on the area of the nuclear plant where they are located.

In the packet header we will include a bit indicating the priority of the message. When sensors detect the RPAS in its transmission range, the sensors would inform about the number of messages that are in the buffer and their priorities to the RPAS. So the RPAS could in real time intelligently readjust the contact time with each sensor. Thus the RPAS could reduce the possibility of containment of transmission that can occur with the scheme we have today.

Security is also one of the critical points, due to the continuous developments of new attacks and the limited applicable regulations for control. Although the proposed Wireless Sensor Network is vulnerable to malicious attacks, 
authentication techniques are rapidly evolving which encourage the deployment of Wireless Sensor Networks in nuclear plants [26].

\section{Conclusions}

In radiological scenarios crisis, due to lack of connectivity it is essential to have alternative schemes to reschedule the sensing of the ground in real time. Our implementation is based on algorithms that exploit infrastructure nodes (sensors, ground vehicles, etc.) by a carrier of messages (the RPAS) responsible for transmitting the information to the control center.

The interface of the sensor network that we programmed in this research is able to reschedule the flight plan of an unmanned aerial vehicle to collect the largest possible amount of information from both sensors and ground vehicles. The interface was integrated in our simulation environment in which software RPAS components can be developed under scenarios of actual air traffic and automatic reconfigurations as real time flight plans.

\section{Competing Interests}

The authors declare that there is no conflict of interests regarding the publication of this paper.

\section{Acknowledgments}

This research is part of the Project "RPAS Operations in the Single European Sky" TRA2013-45119-R partially funded by the Spanish Ministry of Education and Science.

\section{References}

[1] F. E. Schneider, B. Gaspers, K. Peräjärvi, and M. Gårdestig, "Current state of the art of unmanned systems with potential to be used for radiation measurements and sampling: ERNCIP thematic group Radiological and nuclear threats to critical infrastructure Task 3 deliverable 1," 2015.

[2] G. F. Griffin, "The use of unmanned aerial vehicles for disaster management," Geomatica, vol. 68, no. 4, pp. 265-281, 2015.

[3] C. A. F. Ezequiel, M. Cua, N. C. Libatique et al., "UAV aerial imaging applications for post-disaster assessment, environmental management and infrastructure development," in Proceedings of the International Conference on Unmanned Aircraft Systems (ICUAS '14), pp. 274-283, May 2014.

[4] H. Balta, G. De Cubber, Y. Baudoin, and D. Doroftei, "UAS deployment and data processing during the Balkans flooding with the support to Mine Action," in Proceedings of the 8th International Advanced Robotics Programme (IARP) Workshop on Robotics for Risky Environment (RISE), pp. 28-29, 2015.

[5] S. M. Adams, M. L. Levitan, and C. J. Friedland, "High resolution imagery collection for post-disaster studies utilizing unmanned aircraft systems (UAS)," Photogrammetric Engineering \& Remote Sensing, vol. 80, no. 12, pp. 1161-1168, 2014.

[6] A. M. Ambrose-Thurman, Autonomous, collaborative, unmanned aerial vehicles for search and rescue [Ph.D. thesis], Durham University, 2014.
[7] I. Jawhar, N. Mohamed, J. Al-Jaroodi, and S. Zhang, "A framework for using unmanned aerial vehicles for data collection in linear wireless sensor networks," Journal of Intelligent \& Robotic Systems: Theory and Applications, vol. 74, no. 1-2, pp. 437-453, 2014.

[8] M. Abdelkader, M. Shaqura, C. G. Claudel, and W. Gueaieb, "A UAV based system for real time flash flood monitoring in desert environments using Lagrangian microsensors," in Proceedings of the International Conference on Unmanned Aircraft Systems (ICUAS '13), pp. 25-34, IEEE, Atlanta, Ga, USA, May 2013.

[9] S. Kandeepan, K. Gomez, L. Reynaud, and T. Rasheed, "Aerialterrestrial communications: terrestrial cooperation and energyefficient transmissions to aerial base stations," IEEE Transactions on Aerospace and Electronic Systems, vol. 50, no. 4, pp. 2715-2735, 2014.

[10] A. E. A. A. Abdulla, Z. M. Fadlullah, H. Nishiyama, N. Kato, F. Ono, and R. Miura, "An optimal data collection technique for improved utility in UAS-aided networks," in Proceedings of the IEEE INFOCOM, pp. 736-744, IEEE, Toronto, Canada, AprilMay 2014.

[11] M. A. M. Marinho, E. P. De Freitas, J. P. C. Lustosa Da Costa, A. L. F. De Almeida, and R. T. De Sousa, "Using cooperative MIMO techniques and UAV relay networks to support connectivity in sparse Wireless Sensor Networks," in Proceedings of the International Conference on Computing, Management and Telecommunications (ComManTel '13), pp. 49-54, January 2013.

[12] I. Bekmezci, O. K. Sahingoz, and Ş. Temel, "Flying Ad-Hoc Networks (FANETs): a survey," Ad Hoc Networks, vol. 11, no. 3, pp. 1254-1270, 2013.

[13] Z. Gu, Q.-S. Hua, Y. Wang, and F. C. M. Lau, "Reducing information gathering latency through Mobile Aerial Sensor Network," in Proceedings of the 32nd IEEE Conference on Computer Communications (IEEE INFOCOM '13), pp. 656-664, Turin, Italy, April 2013.

[14] D. H. Choi, S. H. Kim, and D. K. Sung, "Energy-efficient maneuvering and communication of a single UAV-based relay," IEEE Transactions on Aerospace and Electronic Systems, vol. 50, no. 3, pp. 2320-2327, 2014.

[15] Plan de Emergencia Nuclear Exterior a las Centrales Nucleares de Ascó y Vandellós, Tarragona (PENTA), BOE num 271, pp. 94293-94361, Novmber 2009, https://www.boe.es/diario_boe/ txt.php?id=BOE-A-2009-17889.

[16] B. Ataul and J. Jin, "A review of wireless sensor network for nuclear power plant application," Nuclear Safety and Simulation, vol. 6, no. 1, 2015.

[17] R. Bogue, "Radiation hardening and sensors for radioactive environments," Sensor Review, vol. 33, no. 3, pp. 191-196, 2013.

[18] "Libelium Comunicaciones Distribuidas S.L. Waspmote Datasheet," http://www.libelium.com/downloads/documentation/ waspmote_datasheet.pdf.

[19] M. Guinaldo, E. Fábregas, G. Farias et al., "A mobile robots experimental environment with event-based wireless communication," Sensors, vol. 13, no. 7, pp. 9396-9413, 2013.

[20] http://www.alphaunmannedsystems.com/products/sniper.

[21] A. Reyes, E. Pastor, C. Barrado, and M. Gasull, "Monitoring radiological incidents through an opportunistic network," IEEE Latin America Transactions, vol. 13, no. 1, pp. 54-61, 2015.

[22] P. Royo, C. Barrado, and E. Pastor, "ISIS+: a software-in-theloop unmanned aircraft system simulator for nonsegregated airspace," Journal of Aerospace Information Systems, vol. 10, no. 11, pp. 530-543, 2013. 
[23] A. Boulis, Castalia, A Simulator for Wireless Sensor Networks and Body Area Networks. Version 3.2. User's Manual, 2011, https://forge.nicta.com.au/docman/view.php/301/592/Castalia +-+User+Manual.pdf.

[24] A. Varga and R. Hornig, "An overview of the OMNeT++ simulation environment," in Proceedings of the 1st International Conference on Simulation Tools and Techniques for Communications, Networks and Systems \& Workshops (Simutools '08), p. 60, ICST (Institute for Computer Sciences, Social-Informatics and Telecommunications Engineering), Marseille, France, March 2008.

[25] ZigBee Alliance. ZigBee Specification, IEEE Standard for Local and Metropolitan Area Networks-Part 15.4: Low-Rate Wireless Personal Area Networks (LR-WPANs), http://www.zigbee.org.

[26] R. I. Gomaa, I. A. Shohdy, K. A. Sharshar, A. S. Al-Kabbani, and H. F. Ragai, "Real-time radiological monitoring of nuclear facilities using ZigBee technology," IEEE Sensors Journal, vol. 14, no. 11, pp. 4007-4013, 2014. 


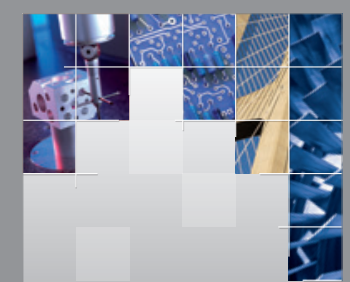

\section{Enfincering}
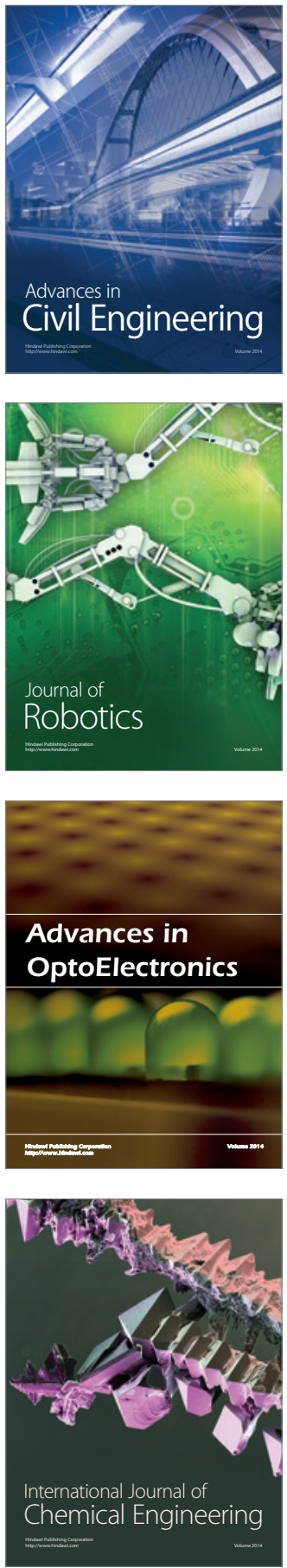

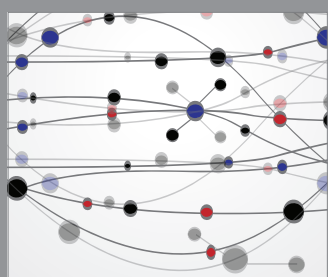

The Scientific World Journal

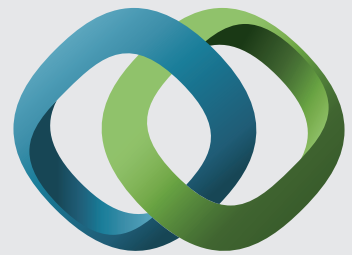

\section{Hindawi}

Submit your manuscripts at

http://www.hindawi.com
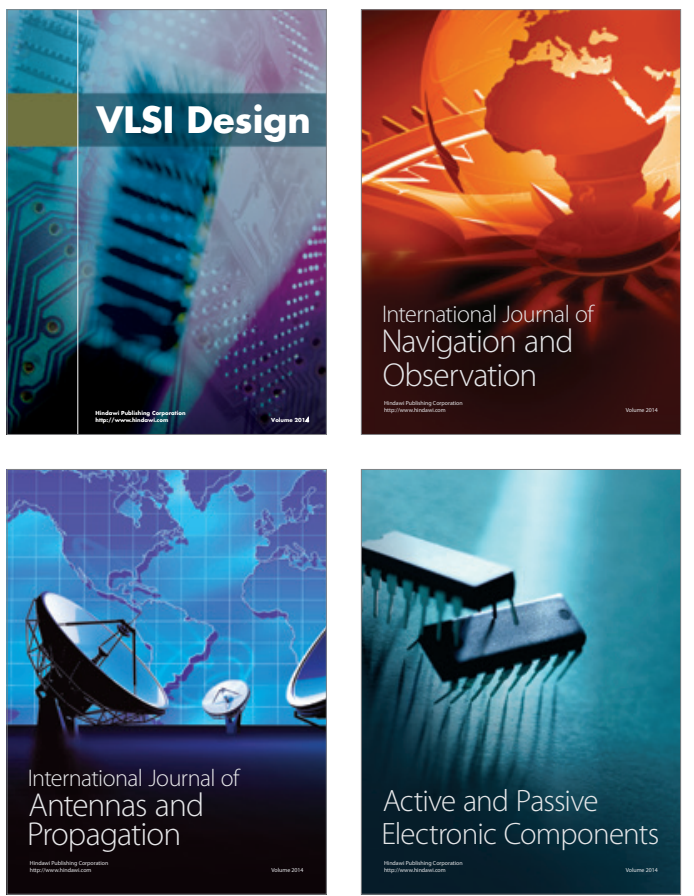
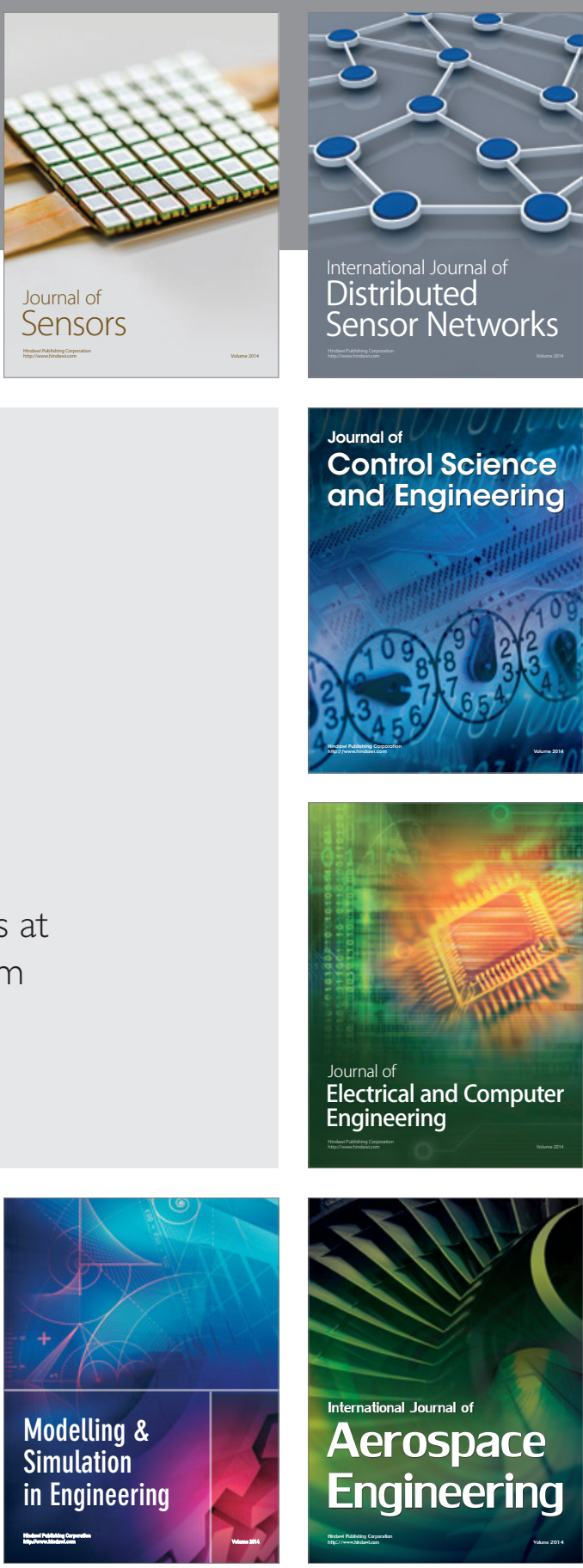

International Journal of

Distributed

Sensor Networks

Journal of

Control Science

and Engineering
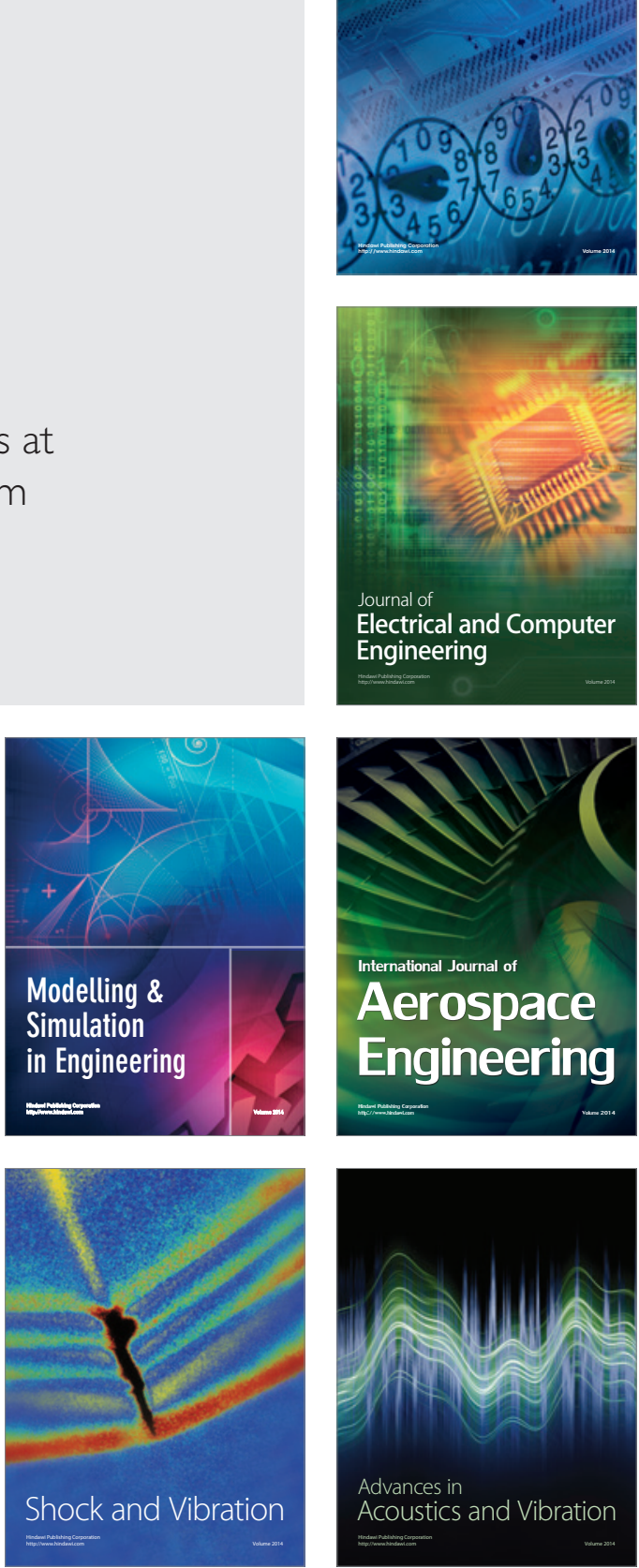